\title{
An Instrument to Study the Diffuse EUV Astronomical Background
}

\author{
S. BOWYER, ${ }^{1}$ J. EDELSTEIN, ${ }^{1}$ M. LAMPTON, ${ }^{1}$ L. MORALES, ${ }^{2}$ \\ J. PEREZ MERCADER, ${ }^{3}$ AND A. GIMENEZ ${ }^{3}$ \\ ${ }^{1}$ Center for EUV Astrophysics, 2150 Kittredge St., \\ University of California, Berkeley, CA 94720-5030, USA \\ ${ }^{2}$ Universidad Autonoma de Madrid, Ciudad Universitaria de Cantoblanco, \\ 28049 Madrid, Spain \\ ${ }^{3}$ LAEFF, Instituto Nacional De Tecnica Aerospacial, \\ Apartado de Correos 50727, 28080 Madrid, Spain
}

\begin{abstract}
The extreme ultraviolet (EUV) diffuse background is the most poorly known of any of the diffuse astronomical backgrounds. Only upper limits to this flux exist, obtained with spectrometers with very crude (from $\approx 15$ to $30 \AA$ ) resolution; these limits are generally one to two orders of magnitude larger than the expected sources of cosmic flux. A variety of source mechanisms have been postulated to radiate in this bandpass; the most discussed is the hot phase of the interstellar medium. A speculative possibility is that hot dark matter in the form of massive, radiatively unstable neutrinos in our Galaxy will produce a unique line in this bandpass. We describe an instrument employing a new type of spectrometer which will provide $\sim 5 \AA$ resolution and unprecedented sensitivity for diffuse EUV radiation. The instrument will be carried aboard the newly developed Spanish Minisat satellite.
\end{abstract}

\section{Introduction}

Initial investigations of the diffuse astronomical background in the EUV were carried out with broad-band detectors on rockets and short duration orbital flights (Cash, Malina, \& Stern 1976; Stern \& Bowyer 1979; Bloch et al. 1986). The Alexis Satellite developed by the Los Alamos group was designed to provide broad band measurements of the cosmic EUV flux (Bloch et al.); and Lieu et al. (1995) obtained upper limits to this flux with EUVE.

A few spectrographic measurements have been made. Holberg (1986) obtained data from 520 to $1100 \AA$ with $30 \AA$ resolution with the Voyager 2 ultraviolet spectrometer. Edelstein \& Bowyer (1995) have noted that the Voyager upper limits were overly stringent and have derived more appropriate limits from this data. Labov \& Bowyer (1991) flew a grazing incidence spectrometer to measure the cosmic background from 80 to $650 \AA$ with a resolution of $15 \AA$ on a sounding rocket. These authors tentatively identified features which might have been produced by the hot phase of the ISM, but the features noted were close to the limiting sensitivity of the instrument and are now believed to be spurious (Edelstein \& Bowyer 1995). Jelinsky et al. (1995) made innovative use of the spectrometers on $E U V E$ to obtain astronomically important upper limits to the EUV background. The Wisconsin group has developed an instrument to carry out soft X-ray spectroscopy on the diffuse cosmic background; preliminary results from this experiment are presented elsewhere in this Volume.

\section{Diffuse Cosmic Emission Mechanisms}

It has been more than thirty years since Spitzer (1956) suggested that hot, milliondegree gas pervades our Galaxy and more than twenty years since the soft X-ray background (now generally believed to be produced by a high-temperature component of the 
ISM) was first detected (Bowyer, Field, \& Mack 1968). However, models ascribing this emission to a high temperature plasma have been surprisingly unsuccessful (Cox 1995). If the diffuse soft X-ray background is the product of emission from a hot gas, much of the radiated power will be in emission lines from highly ionized atoms radiating at EUV wavelengths.

The actual lines observed from a hot ISM will be strongly dependent upon the temperature and thermal history of this material (Breitschwerdt \& Schmutzler 1994). Several temperatures have been suggested for this phase. Soft X-ray data suggest $10^{6} \mathrm{~K}$ gas (Cox \& Reynolds 1987). Absorption line data showing O VI (Jenkins 1978a,b) is often cited in combination with the soft X-ray data as further evidence for a $10^{6} \mathrm{~K}$ gas, but the peak of the emission curve for $\mathrm{O} V I$ is at the substantially lower temperature of $\sim 3 \times 10^{5}$ K. High ionization absorption lines observed in stellar spectra taken with IUE indicate a temperature of $3 \times 10^{5} \mathrm{~K}$ (Savage 1987) as does the observation of emission lines at far UV wavelengths (Martin \& Bowyer 1989). Breitschwerdt \& Schmutzler (1995) have suggested that the soft $\mathrm{X}$-ray emission is the product of residual high ionization states and that the actual kinetic temperature of this plasma could be as low as $4 \times 10^{4} \mathrm{~K}$.

A speculative possible contributor to the cosmic EUV background is emission from neutrinos in our Galaxy undergoing radiative decay. This scenario has been explored in substantial detail in a series of papers by Sciama and co-workers (Sciama 1994). Evidence for this emission has been searched for but not found in IUE data taken on a quasi-stellar object which lies in a cluster of galaxies (Fabian, Naylor, \& Sciama 1991), and with the Hopkins Ultraviolet Telescope, which observed a cluster of galaxies on the flight of ASTRO I (Davidsen et al. 1991). In both cases the line would have been sufficiently redshifted to move it into the bandpass observed. Though these results have been generally interpreted as ruling out the Sciama hypothesis, Bowyer et al. (1995) have shown that this is not correct and that Sciama's hypothesis is still viable.

\section{The Instrument}

We have developed an instrument to measure the diffuse EUV background. We have employed normal incidence rather than grazing incidence optics in this instrument because this allows a folded light path and a much smaller instrument size. Through the use of special coatings, this instrument reaches wavelengths as short as $350 \AA$.

A spectrometer for diffuse radiation disperses radiation viewed through an aperture to a single location on the detector corresponding to the wavelength of the radiation. The observed intensity depends on the product of the area of the aperture times the solid angle of the sky observed. Increasing the width of the aperture increases this product but will degrade the overall spectral resolution. Increasing the solid angle by increasing the aperture height will also increase this product, but grating aberrations in existing spectrograph designs increase rapidly for off-axis rays, strongly limiting the extent to which this parameter can be increased. For example, a conventional Rowland spectrometer performs well for point sources on axis, but has severe aberrations for radiation as little as $1^{\circ}$ off-axis.

To determine potential combinations of grating surface and ruling parameters for an optimum diffuse radiation spectrometer, we developed a general expression describing the optical path for radiation incident upon an arbitrary polynomial surface with variable space diffraction rulings converging to a single point on the detector. We chose planecylindrical radiation emanating from a slit aperture as a source. In contrast, the Rowland spectrograph utilizes a spherical source from a point on the slit. Following Fermat's Principle, we minimized variation of the path function over the grating's aperture and found solutions which eliminated aberrations to third order for on-axis illumination and 


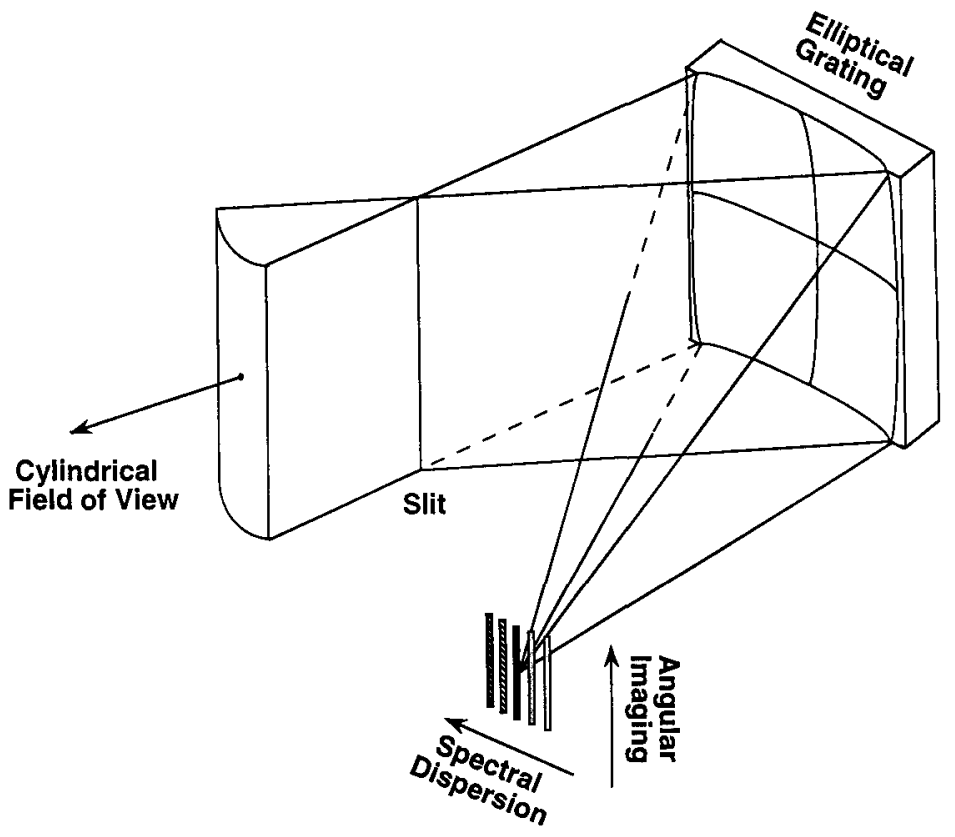

FIGURE 1. Schematic diagram of the optical design of the spectrometer.

retained most of this performance well off-axis. Constant line spacing and rotationally symmetric grating surfaces were then imposed on our solution to simplify the ruling and figuring process, and approximations to the optimum theoretical solution were sought. An elliptical surface was found to provide a solution close to theoretical and this was numerically raytraced over a range of illumination angles and wavelengths to verify the predicted parameters. This design retained at least $80 \%$ of its performance to $4^{\circ}$ off axis. Moreover, spatial resolution better than $0.1^{\circ}$ is achieved along the sky in the direction along the spectrograph slit, which permits radiation from bright stars to be identified and removed. A schematic of the optical design is shown in Figure 1.

A major innovation is the use of a low internal background multichannel plate detector used in connection with wedge and strip encoding (Lampton et al. 1986). At EUV wavelengths a prime contributor to the noise is background in the detector (Lieu et al. 1993). This background consists of two components (a) an internal background due to the radioactive decay of potassium in the microchannel plates, and (b) a charged particle background. We employ special microchannel array plates with no potassium and surround the detector with a charged particle anticoincidence system to significantly reduce both of these backgrounds.

Two of these spectrometers will be flown as a single instrument package (the Espectrografo Ultravioleta extremo para la observacion de la Radiacion Diffusa, or EURD) on the Spanish Minisat satellite in 1996. The gratings on these spectrographs have different 
TABle 1. Key Instrument Parameters

$\begin{array}{ll}\text { Bandpass: } & 350-1100 \AA \\ \text { Field of view: } & 26^{\circ} \times 8^{\circ} \\ \text { Grating: } & 8 \mathrm{~cm} \text { diameter } \\ & 18 \mathrm{~cm} \text { focal length } \\ & \text { holographically ruled } \\ & 2460 \text { lines mm } \mathrm{mm}^{-1} \\ \text { long wavelength: silicon carbide } & \text { short wavelength: boron carbide } \\ \text { Multichannel plate with } & \\ \text { Grating overcoating: } & \text { wedge and strip encoding } \\ & \text { magnesium fluoride } \\ \text { Detector: } & 40 \times 40 \times 13 \mathrm{~cm} \\ \text { Detector photocathode: } & 11 \mathrm{~kg}\end{array}$

coating to optimize the shorter and longer bandpasses of the total bandpass covered. A summary of key instrument parameters is provided in Table 1.

\section{Flight Electronics and Software}

The first stage detector electronics consists of three ultra low noise charge sensitive amplifiers which receive the microchannel plate signals and provide shaped pulse signals to the downstream analog-to-digital conversion system. These charge amplifiers are especially designed to be free of overload saturation artifacts from cosmic-ray events, and are designed to provide image stability over the three year duration of the mission.

An important part of the detector electronics is an electronic pulse calibration system. Each second, a trio of charge pulses is generated by an onboard quartz-crystal controlled oscillator. The amplitudes of these pulses are controlled by a digitally switched attenuator to produce accurate charge signals for the detector electrodes; these charge amplitude ratios have been chosen to encode positions in the extreme corners of the field of view of each detector. In this way, the stability of the entire detector electronics can be monitored through instrument development, calibration, test, integration, and during the mission.

The digital flight electronics system is centered in a high capability microprocessor with associated ROM, RAM, control logic, and communications chips. The architecture adopted is based on the flight proven ATT DSP32C microprocessor. Although this chip family is radiation tolerant, specific provisions have been taken to assure its survival in case of latchup triggered by a high energy cosmic ray event.

There are two principal functions of the EURD microprocessor. A photon formatting task takes random photoevents in their wedge-strip format and converts them into event $(x, y)$ coordinates using full 32-bit arithmetic in order to avoid introducing computational artifacts into the accumulated images. Each event is flagged with the anticoincidence shield status along with a variety of other information.

The data communications task of the EURD microprocessor executes concurrently with the photon formatting. Data are transferred to and from the spacecraft bus in a high-speed block format. The EURD processor is continuously available to receive a command block, or to dispatch a data block to the onboard data storage system.

\section{Overall Performance}

In Figure 2 we compare the predicted sensitivity for the EURD spectrometers for 100 hrs and $1000 \mathrm{hrs}$ of observing time with the best upper limits available in the bandpass 
TABLE 2. Diffuse Galactic ISM Experiments

$\begin{array}{lll} & \text { Bandpass } & \text { Resolution }(\mathrm{E} / \Delta \mathrm{E}) \\ \text { EUVE (Jelinsky et al. 1995) } & 190-250 \AA & 10 \\ & 400-460 \AA & 10 \\ \text { Los Alamos (Bloch et al., 1995) } & 130-190 \AA & 10 \\ \text { Wisconsin (Sanders et al., 1995) } & 40-80 \AA & 20 \\ \text { Penn State (Burrows et al. 1995) } & 10-50 \AA & 40-60 \\ \text { This experiment } & 350-1000 \AA & 200\end{array}$

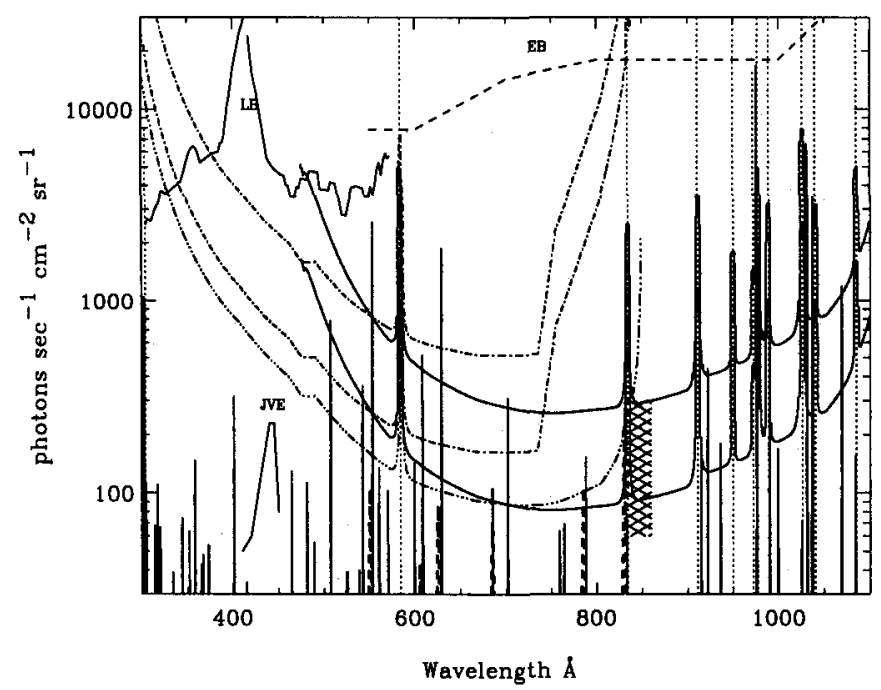

FIGURE 2. Existing upper limits to the diffuse EUV cosmic background. LB are the $15 \AA$ resolution limits of Labov and Bowyer. $\mathrm{EB}$ are the $30 \AA$ resolution limits derived by Edelstein and Bowyer from Voyager data. JVE are the limits of Jelinsky et al. The curved, solid, horizontal lines are the flux limits provided by 100 and $1000 \mathrm{hrs}$ of observation with the long wavelength spectrometer. The curved, dot-dashed, hotizontal lines are the same limits for the short wavelength spectrometer with the aluminum filter. The lower dash-triple dot line show similar limits with no filter. The solid vertical lines are the expected ISM emission from a steady-state collisionally ionized plasma. The heavy dashed vertical lines are the intensities from the delayed recombination model of Breitschwerdt and Schmutzler. The dotted vertical lines are expected airglow lines. The cross-hatched region shows the range of the emission predicted by Sciama for a halo of radiatively decaying neutrinos.

covered. Two distinct models of interstellar line emission are also shown. One model assumes collisional ionization equilibrium, using the emissivities of Monsignori-Fossi \& Landini (1995) and the emission measure from Bowyer et al. (1995). The other model is the delayed recombination model of Breitschwerdt \& Schmutzler (1994 and private communication) whose intensity fits the $0.25 \mathrm{keV}$ soft X-ray background at high galactic latitudes and for which we have assumed attenuation due to the ISM in local cloud of $5 \times 10^{17}$ (Frisch 1994). The line from decaying neutrinos is from Sciama (1994), and the airglow lines are from Chakrabarti et al. (1984). 
In Table 2, we summarize key parameters of instruments which have been, or will soon be used to study the character of the diffuse EUV and soft X-ray background. The EURD instrument has substantial capabilities both in absolute terms and in comparison with these other instruments.

We thank Eric Korpela for help in various aspects of this development and for useful discussions. Gerald Penegor, Josef Dalcolmo, Charles Donnelly, and Ray Chung provided important technical contributions. This work was supported by NASA Grant NRG05003-450.

\section{REFERENCES}

Bowyer, S., Field, G., \& MACK, J. 1968, Nature, 217, 32

Bowyer, S., Lieu, R., Smher, S. D., Lampton, M., \& Knude, J. 1995, Nature, 375, 212

Bloch, J. J., Jahoda, K., Juda, M., McCammon, D., Sanders, W. T., \& Snowden, S. L. 1986, ApJ, L, 308, L59

BLOCH, J. J. 1995, This volume

BreitschwerdT, D. \& Schmutzlen, T. 1994, Nature, 371, 774

BreitschwerdT, D. \& Schmutzler, T. 1995, IAU Symposium 171: New Light on Galaxy Evolution, Heidelberg, ed. Ralf Bender \& Roger Davies, Dordrecht: Kluwer Academic Publishers

BURRows, ET AL. 1995, SPIE Proceedings Volume 2518: EUV, X-Ray \& Gamma-Ray Instrumentation for Astronomy VI, submitted

Cash, W., Malina, R., \& Stern, R. 1976, ApJL, 204, L7

Chakrabarti, S., Kimble, R., \& Bowyer, S. 1984, J. Geophys. Res., 89, 5660

Cox, D. P. \& ReYNolds, R. J., 1987, Ann. Rev. A\&A, 25, 303

Cox, D. P. 1995, these proceedings

DAVIDSEN, A., ET AL. 1991, Nature, 351, 128

Edelstein, J. \& Bowyer, S. 1995, ApJ, submitted

Fabian, A. C., Naylor, T., \& Sciama, D. W. 1991, MNRAS, 249, 21

Frisch, P. 1994, Science, 265, 1423

Holberg, J. B. 1986, ApJ, 311, 969

Jelinsky, P., VAllerga, J. V., \& Edelstein, J. 1995, ApJ, 442, 653

JENKINS, E. B. $1978 \mathrm{a}$, ApJ, 219, 845

JENKINS, E. B. 1978b, ApJ, 220, 107

LABOV, S. E. \& BoWYER, S. 1991, ApJ, 371, 810

Lampton, M., Siegmund, O. H. W., Bixler, J., \& Bowyer, S. 1986, Proc. SPIE, 627, 383

LeU, R., Bowyer, S., Lampton, M., Jelinsky, P., \& Edelstein, J. 1993, ApJ, 417, L41

MARTIN, C. \& BoWYER, S. 1989, ApJ, 338, 677

MONSIGNORI-FossI, B. \& LANDINI, M. 1995, these proceedings

SANDERS, W. T. \& EDgar, R. J. 1995, these proceedings

SAVAge, B. D. 1987, in Interstellar Processes, ed. Hollenbach \& Thronson, Dordrecht: Reidel Publishing

Sciama, D. W. 1994, Modern Cosmology and the Dark Matter Problem, New York: Cambridge University Press

SPITZER, L., JR. 1956, ApJ, 124, 20

STERN, R. \& BOWYER, S. 1979, ApJ, 230, 755 